\title{
XVIII.
}

\section{Ueber das Verhalten der Nervenkörperchen in kranken Nerven.}

\author{
Von \\ Prof. Dr. Albert Adamkiewicz \\ in Krakau. \\ (Hierzu Taf. XII.)
}

Als ich vor nunmehr drei Jahren in den peripherischen, speciell doppelt contourirten und markhaltigen Nervenfasern des erwachsenen Menschen vorher nie gesehene zellige Elemente, meine „Nervenkörperchen “*) beschrieb, - da warde auch ihnen jenes Schicksal zu Theil, das so viele neve Thatsachen auf wissenschaftlichem Gebiete trifft: Man leugnete zuerst ihre Existenz, dann schrieb man sie einem früheren Autor zu und endlich gab man ihnen einen fremden Namen und führte sie so von Neuem in die Wissenschaft ein.

Vignal**) war es, der meine Nervenkörperchen nicht hat darstellen können und sie für einfache Gerinnungen in der Schwannschen Scheide erklärte. - Benda ${ }^{* * *}$ ) meinte, meine Nervenkörperchen seien die bereits von Ranviert) beschriebenen noyaux du ségment inbrannulaire und Rosenheim + ) hielt es für gut, meine Nervenkörperchen als „Mastzellen“ zu bezeichnen, mich in der Folge

*) Sitzungsbericht der k. Akademio der Wissenschaften in Wien 1885. Bd. 91 and 1888 . Bd. 97.

**) Compt. rend. de la soc. de biol. Paris 12. Mars 1886.

***) Verhandl. d. physiol. G. zu Berlin. 1886. No. 17 und 18.

f) Vergl. meine oben eitirte Arbeit,

†) Vergl. V'erhandl. der physiol. G. zu Berlin. 1886. No. 17 und 18 and Dieses Auchiv XVII. 820. 
aus der Discussion ganz zu eliminiren und seine Mastzellen für Elemente des Bindegewebes in Nerven, speciell für pathologische Bildungen, Producte der Neuritis, auszugeben.

Die Vignal'sche Ansicht konnte sich der Thatsache gegenüber nicht lange halten, dass Zellen mit Kern und Protoplasma - keine Gerinnungsproducte sind. - Benda's Vermuthung wurde dadurch erledigt, dass Ranvier*), der meine Präparate, zu sehen die Güte hatte, freimüthig erklärte. nichts meinen Nervenkörperchen Aeholiches vorher je beobachtet zu baben. - Und bezüglich der Mastzellentheorie Rosenheim's wurde in meinem Laboratorium der sorgfältigste Nachweis geführt ${ }^{* * *}$ ), dass dieselben weder der Form, noch den Tinctionsverhältnissen, noch dem Vorkommen nach irgend etwas mit den Nervenkörperchen Gemeinsames haben.

So durfte die Existenz der Nervenkörperchen als eigenartiges Gebilde vorläufig sichergestellt angesehen werden. - Und es war demnächst zu entscheiden: 1. ob sie Elemente der gesunden Nerven seien, - und 2. wie sie sich in kranken Nerven verhielten.

1. Fasse ich alles bezüglich des ersten Punktes über die Nervenkörperchen bisher Festgestellte kurz zusammen, so hat sich Folgendes ergeben:

Die Nervenkörperchen sind spindelförmige, an den Enden zugespitzte Zellen von halbmondförmigem Querschnitt, ovalem Kern in der Mitte and reichem Protoplasma an beiden Enden. Durch Safranin wird jener violett, dieses orange gefärbt. Die Nervenkörperchen sitzen zwischen Mark und Schwann'scher Scheide und kommen in allen doppeltcontourirten und markhaltigen Nervenfasern des erwachsenea llenschen ungefähr vom 10. Jahr bis an das Lebensende immer in derselben Menge vor. In den motorischen Nerven sowohl den cerebrospinalen, als den cerebralen sind sie besonders zahlreich und in grossen Exemplaren vertreten.

Den Nervenkernkörperchen analoge, ja auch nur ähnliche Gebilde sind, soweit meine bisherigen Erfahrungen reichen, ausser an den angeführten Orten nirgends anzutreffen. Sie fehlen, namentlich auch in den Nerven des Thieres und selbst, wie erwähnt, beim Menschen in den ersten Jahren des Lebens.

In der letzten Zeit habe ich, - was gewiss von besonderem anthropologischem Interesse ist, - auch die Nerven des Affen vergeblich auf Nervenkörperchen untersucht. Und so darf wohl behauptet

*) Vergl. meine oben citirte Arbeit.

**) Momidlowski, Wiener klinische Wochenschr. 1888. No. 19 a. 20. 
werden, dass die Nervenkörperchen ein physiologischer Bestandtheil der doppelt contourirten, markbaltigen, zumal motorischen Nervenfasern und zugleich die einzigen morphologischen Elemente sind, welche, wie es wenigstens bis jetzt scheint, dem erwachsenen Menschen ganz ausschliesslich zukommen.

2. Die nächste Frage, die uns nach der Feststellung der physiologischen Verhältnisse der Nervenkörperchen interessiren muss, ist, wie erwähnt, die nach dem Verhalten derselben in kranken Nerven.

Aber es stehen der Lösung dieser Frage grosse Schwierigkeiten entgegen.

Denn die Nervenkörperchen kommen bei Thieren nicht vor. Und diese Thatsache beraubt uns eines Mittels, welches uns der Lösung der gestellten Frage am schnellsten näher bringen würde, - der Möglichkeit, dieselbe durch das Experiment zu lösen.

So bleiben wir auf die Untersuchung kranker Nerven vom kranken Menschen angewiesen. Und was ich an solchen bisher habe feststellen können, ist in Kürze folgendes:

Einem Fall von sogenannter „Pachymeningitis hypertrophica“, über den eine andere Arbeit*) nähere Details gebracht hat, entnahm ich das zur Untersuchung nöthige Material an kranken Nerven.

Wir verstehen seit Charcot unter Pachymeningitis hypertrophica jenen krankhaften Zustand der Rückenmarkshänte, in Folge dessen letztere durch mächtige Bindegewebsneubildungen sich verdicken und gleichzeitig unter einander und mit der Nachbarschaft verwachsen. In den allerschwersten Fällen dieser Krankheit schmelzen dann Wirbelkanal, Rückenmarkshäute und Rückenmarksubstanz in Eins zusammen.

Indem dieser bindegewebige Kitt, diese zur Retraction neigende Narbenmasse der Schwarte die sonst freien und nur durch die Rückenmarkshäute getheilten, zwischen Rückenmark und Wirbelkanal befindlichen Räume durchwuchert, drückt, ja umschnürt sie die unter normalen Verhältnissen jene Räume allein durchziehenden Nervenwurzeln, stört sie mehr oder weniger in ihrer Ernährung und macht sie auf diese Weise krank. Und weil bei so massenhafter Bindegewebsumwucherung die Wirkungen derselben auf die Nerven an verschiedenen Orten dem Grade nach sehr variiren, - so können wir auch bei

*) Die Pachymeningitis hypertrophica und der chronische Infaret des Rüokenmarkes. Monographie. Wien, 1890. Hölder. 
schwereren Formen von chronischer Pachymeningitis den verschiedensten Stadien und Abstufungen von Nervenentartung begegnen und damit ein ausreichendes Material zur Beurtheilung des Antheils gewinnen, welchen die Nervenkörperchen am pathologischen Zustande ihrer Nerven nehmen.

Die Schwarte war in unserem Fall am stärksten entwickelt am Halsmark, wo ihre Dicke einige Millimeter betrug. Nach unten zu nahm sie an Stärke ab und endete in Gestalt gröberer und schwächerer Verwachsungen im mittleren und unteren Brustmark.

Dem entsprechend gab es die meisten umschnürten und degenerirten Nerven in den Nervengeflechten des Halses, weniger kranke Nerven in den tiefer gelegenen Plexus des Brustmarkes und gar keine im Plexus cruralis, der ganz und gar gesund war.

Es konnte unter solchen Umständen nicht fehlen, dass in den von der pachymeningitischen Schwarte umwucherten Rückenmarkswurzeln sich auch sehr viele Uebergänge von gesunden zu vollkommen entarteten Nerven befanden.

Da aber die hypertrophische Pachymeningitis mit ihren Gewebsneubildungen ein exquisit chronischer Process ist, der auch in den von ihr ergriffenen Nerven nur langsam fortschreitende Entartungsvorgänge wachrufen kann, so musste von vornherein darauf verzichtet werden, in den kranken Nerven diejenige Form der Degeneration zu finden, welche die acute Entartung charakterisirt, nach plötzlichen Trennungen der Nerven von ihren ernährenden Centren entsteht und durch den bekannten Zerfall der Markscheide charakterisirt ist.

In welcher Weise sich die Nervenkörperchen an solchem acuten Zerfall der Markscheide betheiligen, - das ist demnach eine Frage, auf welche zu antworten ich mir vorläufig versagen muss. Während wir dagegen von dem uns zu Gebote stehenden eben erwähnten Material Aufschluss über dasjenige Verhalten der Nervenkörperchen erwarten durften, welches sie in chronisch degenerirenden, demnach langsam zu Grunde gehenden Nerven zeigen.

3. Man kann die chronische Degeneration der Nerven kurz als eine „Atrophie“, einen progressiven Schwund derselben bezeichnen. Es ist selbstverständlich, dass dieser Schwund eine ganze Skala von Veränderungen durchläuft, bevor er an sein durch vollständigen Untergang der Nervensubstanz gekennzeichnetes Endziel anlangt.

Zur Aufklärung der uns interessirenden Frage wird es genügen, Anfang, Ende und ein mittleres Stadium dieser Degeneration zu unter- 
scheiden und den Antheil der Nervenkörperchen an diesen drei Stadien des Nervenschwundes festzustellen.

Es wurde bereits hervorgehoben, dass die Nervenkörperchen zu den motorischen Nerven in besonders enger Beziehung stehen. Wir wollen uns deshalb bei der Lösung unserer Aufgabe an die kranken vorderen Wurzeln halten und zunächst ein gesundes Nervenbündel betrachten, damit wir auf dessen Grunde die pathologischen Abweichungen wie auf einer Folie mit um so grösserer Schärfe erkennen.

Das Verhalten der Nervenkörperchen im normalen Nerv - und diesen selbst habe ich nach dem Ergebniss der Tinction mit Safranin bereits des Genaueren geschildert*). Indem ich auf diese Schilderung verweise, möchte ich hier nur kurz hervorbeben, dass für den normalen motorischen Nerv (vergl. die Zeichnung auf Taf. XII, Fig. I, A) charakteristisch sind: eine dünne (violett gefärbte) Hülle ( $P$ - Perineurium) von gewöhnlichem, fibrillärem Bindegewebe mit ovalen Kernen, ein sehr feines (gleichfalls violettes) Netz von Fäden, das vom Perineurium ausgehend, das Innere des Nerven in enge, gleichmässige Maschen theilt und mit runden Kernen durchsetzt ist (E - Endoneurium), - grosse markreiche (orange gefärbte) Nervenfasern (N), die locker in diesen Maschen liegen und zumal aus dünnen Schnitten schon bei l̨leichtem Zerren oder Drücken der Präparate fallen, und endlich die branen Halbmonde der (quer durchschnittenen) Nervenkörperchen (Nk), die gross und stattlich über den Nervenquerschnitt zerstreut sich an einzelne Nervenfasern schmiegen.

4. Die pathologischen Abweichungen von diesem Bilde fallen sofort in die Augen.

a) Ihm am nächsten stehen Präparate, in denen die einzelnen Elemente des Nervenbündels zwar dieselben sind, wie in gesunden Nerven, - aber zum Theil bereits verändert erscheinen (Fig. I, B).

Es gilt das nicht von den Elementen des Peri- und des Endoneuriums, die noch vollkommen normal sind, sondern ausschliesslich von den Nervenfasern (N, Fig. I, B) und von den Nervenkörperchen selbst (NK, Fig. I, B).

Vor allem fällt an den Nervenfasern die Kleinheit ihrer Dimensionen und die Blässe ihrer Färbung auf. Ihr Querschnitt ist im Vergleich zu normalen Nerven deutlich reducirt. Und das Safranin färbt sie kaum noch deutlich gelb, lässt sie hier und dort fast farblos

*) Sitzungsber. der k. Akad. der Wissensoh. zu Wien. Bd. 97. 1888. 
oder grau erscheinen. An den Nervenkörperchen macht sich mehr ihre Kleinheit, weniger die Farbenschwäche bemerkbar (Fig. I, B). Sie sind meist kaum halb so gross, als im normalen Zustande; aber immer noch von ziemlich gesättigter orange-gelber Farbe.

Es handelt sich hier offenbar um das erste Stadium der Krankheit, ein Stadium, das sich demnach auf die Nervenfasern beschränkt und sich scharf und klar als eine Atrophie derselben, speciell ein Schwund ihrer Markscheiden kennzeichnet. Und dieser Schwund findet in der Abnahme sowohl der Masse, als der Tinctionsfähigkeit der Markscheiden ihren Ausdruck.

Während aber, wie erwähnt, die bindegewebigen Elemente des Nervenbündels an der Krankheit der Nervenfasern in diesem Stadium keinen Antheil nehmen und sich von dem Verhalten in gesunden Nervenbündeln nicht unterscheiden, - erkranken mit den Nervenfasern zugleich auch die Nervenkörperchen, indem auch sie an Masse und an Tractionsvermögen Einbusse erleiden.

Haben wir demnach in den geschilderten Veränderungen des Nervenbündels Anfänge des progressiven Nervenschwundes vor uns, so können wir dieselben kurz dahin definiren, dass sie auf einer Atrophie der Markscheiden beruhen, an denen sich die Nervenkörperchen in unzweideutigster Weise mit ihrer Atrophie betheiligen.

b) Die meisten Nerven aber zeigen weitaus andere Verhältnisse.

Sie sind reich an Bindegewebe und arm an Nervenelementen geworden. Vergl. Fig. II.

An Stelle eines zarten sich leicht vom Nervenbündel ablösenden Perineurium ist ein mächtiger Ring (P) von fibrillärem Bindegewebe getreten, der das Bündel fest umschliesst, - eine Art Pachyneuritis. Man erkennt in dieser Masse wellige Fibrillenzüge (p), die von dem Perineurialring ausgehen und ihn an das Innere binden. Der Hauptsache nach aber besteht sie, wie man besonders an Zerzupfungspräparaten erkennt, aus einem Filz von feinen, an runden Kernen reichen Fäden (E f), die den Fibrillen des Endoneuriums gleichen.

In diesem Filz eingemauert und mit ihm unlösbar verwachsen sitzen, - durch krankhaft verdickte Bindegewebsscheiden weit von einander getrennt, - die Nerven.

Sie sind offenbar noch kleiner, als wir sie im ersten Stadium der Degeneration gesehen haben und, was vor Allem auffällt, in weit geringerer Zahl vorbanden als hier und im normalen Nerven.

Die Nervenkörperchen aber entsprechen wiederum dem Verhalten der Nerven. Soweit sie vorhanden sind, sind sie kleiner 
als unter normalen Verhältnissen und zum Theil nur noch in Rudimenten: in Klümpchen, oder schmalen Streifen, vertreten.

Und dann ist ihre absolute Zahl, wie die der Nervenfasern selbst, kleiner geworden. Und daraus ergiebt sich der neue Schluss, dass die Nervenkörperchen nicht nur erkranken, wenn die Nervenfasern der Atrophie unterliegen, sondern auch, dass sie in demselben Verhältniss zu Grunde gehen, als Nervenfasern verschwinden.

c) Die schwersten Veränderungen, die die kranken Nervenbündel aufweisen, lassen sich kurz dahin definiren, dass das Nervenbündel in einen fast soliden Bindegewebsstrang verwandelt erscheint, der nur noch ganz spärliche Nervenreste aufweist (Fig. III.). Das Perineurium $(P)$ ist nunmehr nur noch auf Querschnitten an dem horizontalen Verlauf seiner Fasern kenntlich.

Und an der Stelle des Endoneuriumnetzes und seiner Nerven ist ein nach allen Richtungen sich kreuzendes, in parallele wellige Züge getheiltes reguläres, an ovalen Kernen reiches Fibrillengewebe (E f) getreten.

Dieser fibrilläre Inbalt ist mit dem Perineurium zu einer Masse verwachsen und nur am Verlauf seiner Fibrillen von denen des Perineuriums zu unterscheiden.

Von Nerven aber sind in diesen Bindegewebsbündeln nur noch sehr spärliche Reste vorhanden; hier und dort eine Faser, klein und schattenhait, wie etwas Fremdartiges in das Bindegewebe eingesprengt. Aber wo sie zu erkennen sind, da ist meist auch der ockerfarbige Fleck, - ein brannes Klümpchen als letzter Ueberrest des einstigen Nervenkörperchen sichtbar.

5. Wir köunen vorstehende Ergebnisse folgendermassen zusammenfassen:

Durch Wucherungen im Bereich der Rückenmarkshänte ist in den Nerven der Rückenmarkswurzeln ein Krankheitsprocess erregt worden, den zwei Vorgänge charakterisiren, - Untergang von Nervensubstanz, Neubildnng von Bindegewebe.

Sicher ist, dass diese beiden Processe mit einander Hand in Hand gehen, da in den einzelnen Nervenbündeln um so mehr Bindegewebe vorhanden ist, je weniger Nerven in denselben übrig geblieben sind und umgekehrt. - Aber mit weniger Gewissheit wird festznstellen sein, in welcher causalen Beziehung diese beiden Processe zu einander stehen, - ob die Nervenatrophie die Bindegewebsentwickelung, oder diese den Nervenschwund herbeigeführt habe. 
Für die erste Auffassung sprechen Präparate, in welchen nur die Nerven krank, das Bindegewebe aber noch gesund ist (Fig. 1, B.), für die zweite diejenigen Nervenbündel mit relativ noch gut erhaltenen Nervenfasern, welche von einem dicken Bindegewebsring umwachsen und vom fibrillären Bindegewebe durchzogen sind.

Wie dem auch sei, - Thatsache ist, dass, wo in den peripherischen Nerven die Nervenfasern verschwinden, ob primär oder secundär, - die Nervenkörperchen stets deren Schicksal theilen und mit ibnengleichzeitig verkümmern und zu Grunde geben. -

Wenn aber die Nervenkörperchen in engsten Beziehungen zu den Markscheiden stehen, - physiologisch dort am mächtigsten und zahlreichsten sind, wo die Nerven besonders breit sind und ein stattliches Nark besitzen und pathologisch sich eng den Veränderungen der Markscheide anschliessen, - mit ihr verkümmern und ganz verschwinden, keinesfalls aber durch Krankbeiten der Nerven entstehen oder vermebrt werden, - dann wird Niemand mehr bezweifeln können, dass die Nervenkörperchen nicht nur physiologische Elemente der Markscheide sind, sondern auch als Elemente angesehen werden müssen, deren Lebensschicksale von denjenigen der Nervenfaser speciell deren Markscheide $a b$ hängen. 

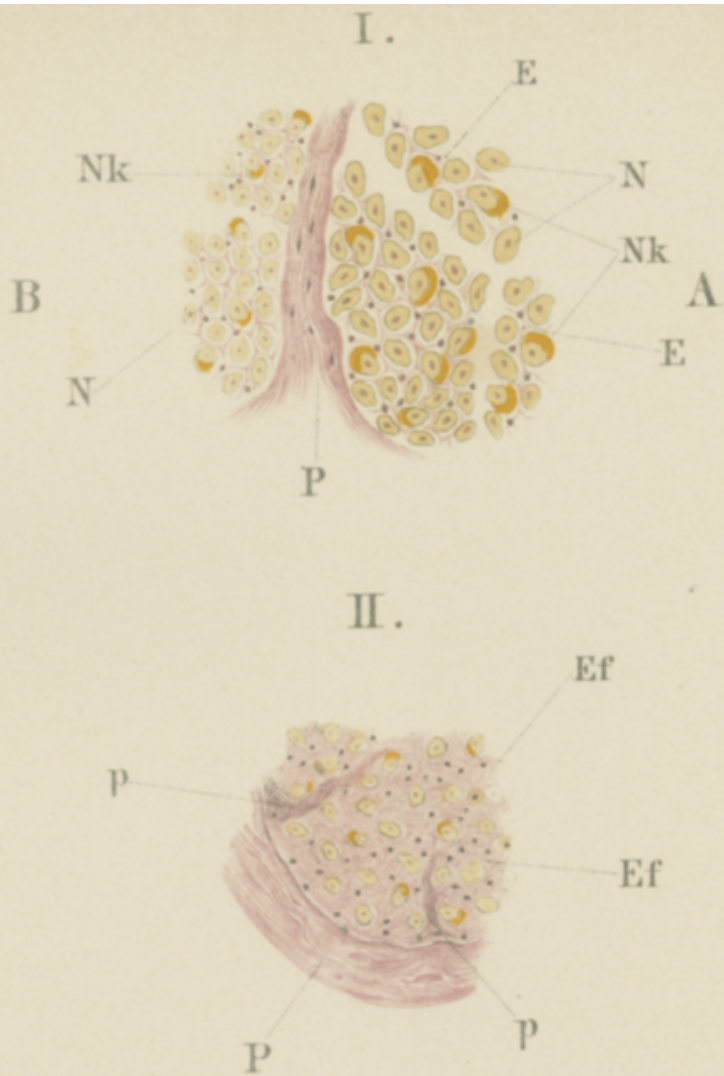

III.

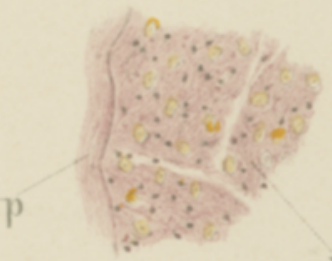

Ef 